\title{
Photodissociation of High Molecular Weight Peptides and Proteins in a Two-Stage Linear Time-Of-Flight Mass Spectrometer
}

\author{
Mary E. Gimon-Kinsel, Gary R. Kinsel,* Ricky D. Edmondson, and David \\ H. Russell \\ Laboratory for Biological Mass Spectrometry, Department of Chemistry, Texas A \& M University, \\ College Station, Texas, USA
}

\begin{abstract}
A two-stage linear time-of-flight mass spectrometer is used to investigate the requirements for performance of laser photodissociation of peptide and protein ions. Results are presented that demonstrate that desorption and dissociation laser pulses can be synchronized to irradiate ions that travel at high velocities down the drift tube of a time-of-flight mass spectrometer. For example, 193-nm photodissociation of bovine insulin and doubly charged lysozyme is demonstrated, and laser power studies suggest that dissociation is initiated by the absorption of a single 193-nm photon. These results are encouraging because they suggest that laser photodissociation of high molecular weight proteins can lead to fragmentation on time scales compatible with time-of-flight mass spectrometry. (J Am Soc Mass Spectrom 1995, $6,578-587)$
\end{abstract}

$\mathrm{M}$ atrix-assisted laser desorption ionization (MALDI) has emerged as a powerful method for the analysis of peptides, proteins, and other classes of polar thermally labile molecules [1]. MALDI allows biomolecules with molecular weights that exceed $100 \mathrm{kDa}$ to be efficiently ionized to form singly or multiply charged intact molecule ions. In addition, the sensitivity of MALDI is excellent; sample sizes range from subfemtomole to picomoles, depending on both the type of analyte and matrix compound used. MALDI is generally regarded as a "soft" ionization method because the abundance of fragment ions in the linear time-of-flight (TOF) mass spectrum is low [2]. Although "soft" ionization is advantageous for molecular weight determination, especially for analysis of individual components of a complex mixture, the presence of fragment ions in the mass spectrum can reveal a wealth of structural information [3]. For example, tandem mass spectrometry has evolved as an important bionanalytical method because it effectively combines the use of "soft" ionization methods and dissociation chemistry for separation of mixtures, molecular weight determinations, and structural information [4]. In most cases tandem mass spectrometry is used for studies of the primary structure of peptides that have molecular weights of 500-2000 Da. Currently there are two limitations to expansion of structural

Address reprint requests to Dr. David $H$. Russell, Laboratory for Biological Mass Spectrometry, Department of Chemistry, Texas A \& M University, College Station, TX 77843-3325.

* Current address: Department of Chemistry and Biochemistry. The University of Texas at Arlington, Arlington, TX 76019-0065. studies to larger peptides and to other classes of biomolecules: (1) the efficiency of collision-induced dissociation (CID) [5], the most common method for performance of structural tandem mass spectrometry studies, is very low for ionic species that have molecular weights of 1500-2500 $u$ and (2) according to statistical theory the rates of dissociation of large ionic species decrease with increasing size, assuming the amount of energy transferred by collisional activation remains constant; thus, reaction products must be collected over a longer reaction time or the internal energies of the ions must be significantly increased.

Activation of ions to produce structural fragmentation can be achieved by methods other than CID, for example, surface-induced dissociation (SID) [6] and photodissociation [7]. Of these latter two methods, pulsed laser photodissociation seems particularly appropriate for activation of ionic forms of large biomolecules because the activation energy is deposited directly into the analyte ion via the absorption of a photon(s) without the need for ion-molecule or ion-surface collisions. Thus, the upper limit of ion activation is set by the available photon sources, for example $118 \mathrm{~nm}(10.5 \mathrm{eV}), 193 \mathrm{~nm}(6.4 \mathrm{eV})$, the common laser wavelengths $266 \mathrm{~nm}(4.7 \mathrm{eV})$ and $355 \mathrm{~nm}$ $(3.5 \mathrm{eV})$, and the photophysics and photochemistry of the ionic system.

Ions produced by MALDI are generally analyzed by time-of-flight mass spectrometry (TOFMS) [8] or Fourier transform ion cyclotron resonance mass spectrometry (FTICR-MS) [9]. Tandem mass spectrometry with laser photodissociation of ions produced by a 
variety of methods has been demonstrated for both TOFMS [10] and FTICR-MS [11]. Of these two techniques, tandem TOFMS offers the advantages of relatively inexpensive and simple instrumentation. Two further considerations are the mechanical complexities of aligning the laser with the ion cyclotron resonance (ICR) ion cell, especially on systems equipped with superconducting magnets, and the vastly different time scales of the ICR and TOF instruments. The ICR photodissociation experiment detects photofragment ions formed over a time interval of milliseconds to seconds, where the TOF photodissociation experiment is limited to detection of photofragments formed on the microsecond time scale. Consequently, these two methods should be viewed as complementary, especially in terms of fundamental studies aimed toward detailed studies of the photochemistry and photophysics of large molecules. On the other hand, laser photodissociation that uses the TOF apparatus is more complicated than for the ICR apparatus. Implementation of tandem TOFMS requires isolation of the analyte ions of interest from other ions produced during the pulsed ionization event, synchronization of the photodissociation laser pulse with the packet of isolated analyte ions, and mass analysis of the resultant photodissociation product ions with sufficiently high spectral resolution to identify the product ion species. With effective solutions to these experimental challenges, laser photodissociation via tandem TOFMS can be applied to fundamental as well as analytical studies of high molecular weight analytes.

Perhaps of more concern in the development of photodissociation of high molecular weight analytes are fundamental questions that surround the ability to activate and dissociate ions that have large numbers of degrees of freedom. Statistical theory, in the form of both quasi-equilibrium theory (QET) and Rice-Ramsperger-Kassel-Marcus (RRKM) theory [12], suggests that dissociation of high molecular weight analytes on a time scale compatible with TOFMS would require unrealistic amounts of activation energy. For instance, recent theoretical calculations by Griffin and McAdoo [13] suggest that $12-19 \mathrm{eV}$ of energy are needed to fragment a $2.1-\mathrm{kDa}$ peptide at rates compatible with TOFMS, and more than $58 \mathrm{eV}$ would be required to fragment a 13.7-kDa protein. Hunt et al. [14] has examined the photodissociation of peptide ions and shown that "structurally significant" fragment ions as well as immonium ions were formed. These experiments were performed with an FTICR-MS, and it is unclear whether the immonium ions were formed by multiphoton processes or by further photodissociation of photofragment ions. McLafferty and co-workers [15] recently reported the infrared multiphoton dissociation (IRMPD) of several electrosprayed peptides and proteins via a continuous-wave $\mathrm{CO}_{2}$ laser and an FTICR. The reported IRMPD photofragment spectra contain structurally significant ions similar to those obtained by CID. Biemann's group [16] also has reported photofragment ion spectra for several peptides and found strong similarities to the CID spectra. Another issue that needs further consideration is the suggestion by Schlag and Levine [17] and Bernshtein and Oref [18] that the dissociations of large molecules involve nonstatistical reactions. Recent studies from a number of laboratories show that abundant fragment ions are formed by both prompt [19] and slow dissociation channels when peptides are ionized by MALDI. Clearly, metastable decay of molecular ions as large as bovine insulin $[\mathrm{M}+\mathrm{H}]^{+}$ions produced by MALDI does occur [20]. Regardless of the implications of these results to the question of statistical or nonstatistical dissociation reactions, the issues of activation of large peptide and protein ions by photon absorption needs to be addressed further and may have important applications. For example, researchers have reported that the efficiency for CID to yield complete sequence information of large ions $\left(M_{\mathrm{r}} \geq 10 \mathrm{kDa}\right)$ formed by electrospray ionization (ESI) is poor [21]. It has been suggested that the CID efficiencies may be charge state dependent [22] and/or there may be competing reaction channels, but other possible explanations such as the influence of the peptide conformation on the available dissociation channels, have not been addressed adequately.

The preliminary MALDI laser photodissociation experiments described in this report are focused on achieving four goals: (1) to show that the experimental methodology for irradiation of high velocity ions as they move down the flight tube of the TOFMS can be implemented, (2) to demonstrate that sufficiently high photon fluxes are available to photodissociate a significant fraction of the irradiated ions, (3) to identify the various signal sources that contribute to the background in the product ion spectrum that results from photodissociation of the intact peptide or protein analyte ions, (4) to determine whether 193-nm photodissociation of high molecular weight proteins, without regard to the types of product ions produced, is feasible and if so, how many photons are absorbed by the analyte prior to the observed ion dissociation.

\section{Experimental}

A schematic of the two-stage acceleration linear TOFMS and peripherals used for the MALDI photodissociation studies is shown in Figure 1. The photodissociation apparatus consists of two linear TOF instruments with dimensions defined by the positions of a series of gridded electrodes. TOF-I corresponds to the $40-\mathrm{cm}$ electrically isolated flight tube liner and TOF-II corresponds to the region between TOF-I and the microchannel plate ion detector. The ion source design has been described previously [23] and the final lens of the ion source makes direct contact (via a spring) with the flight tube liner. The final source lens and the flight tube liner can be operated at potentials between ground and $15 \mathrm{kV}$. Thus, the velocity of the ions in TOF-I is 


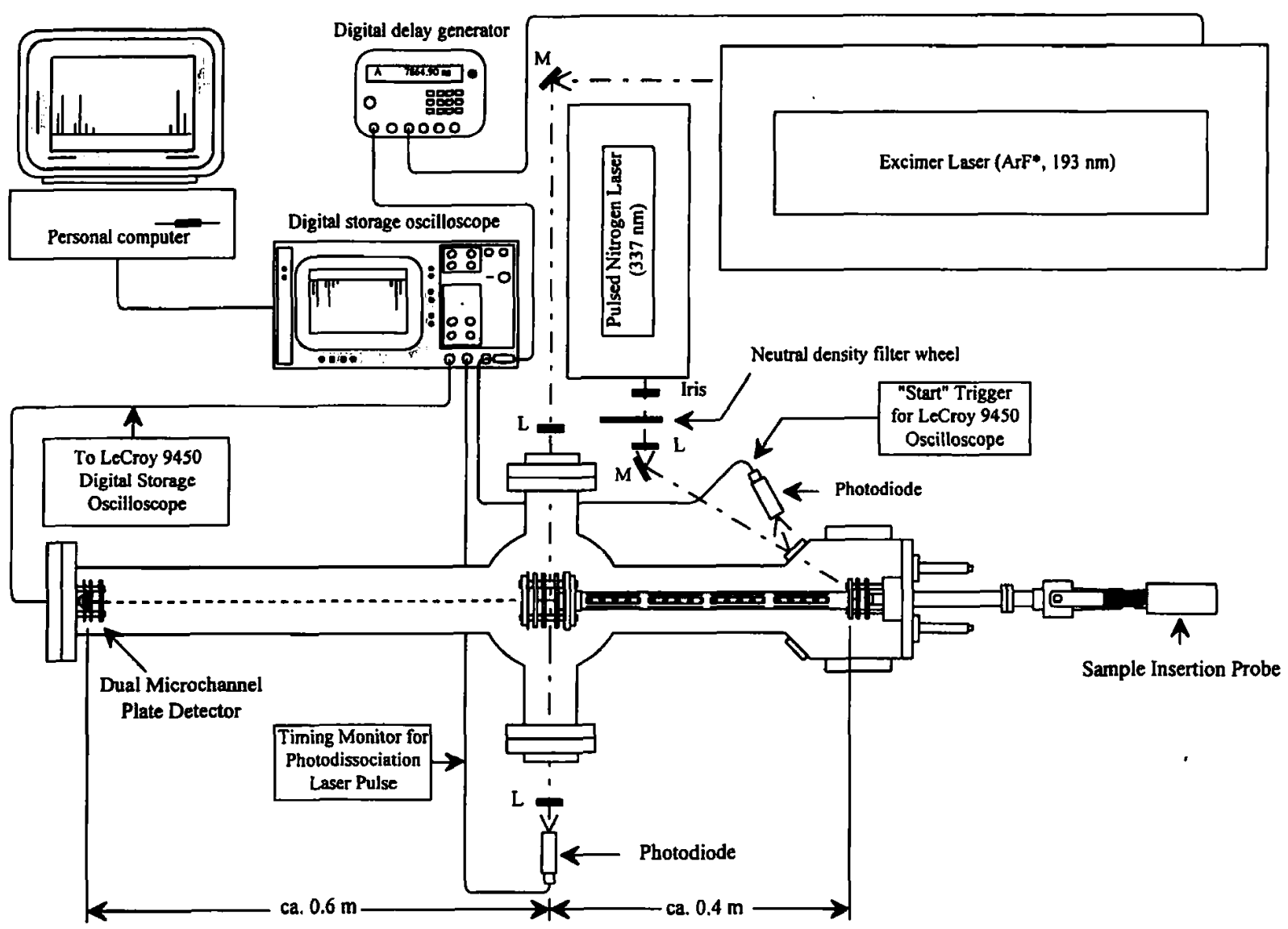

Figure 1. Schematic of the two-stage linear TOFMS instrumentation used for MALDI/193 nm photodissociation studies. See text for description of the experimental details.

determined by the potential difference between the ion repeller and the flight tube liner. The other end of the flight tube liner makes contact (via a second spring) with a second gridded electrode, which defines the entrance to the photodissociation region. A third gridded electrode at a distance of $1 \mathrm{~cm}$ and biased at the same potential as the flight tube, defines the exit of the photodissociation region. Following the photodissociation region exit electrode, at a distance of $0.6 \mathrm{~cm}$, a fourth grounded electrode serves as the entrance to a second $60-\mathrm{cm}$ field-free drift region (TOF-II). A fifth grounded electrode defines the end of TOF-II. A dual microchannel plate detector is positioned $0.6 \mathrm{~cm}$ behind the final grid and is biased at $-2.25 \mathrm{kV}$. Operation of the instrument as a tandem TOF photodissociation apparatus is rather straightforward. Positive ions produced by $337-\mathrm{nm}$ MALDI are accelerated from the source region into the first field-free drift region where individual isomass ion packets separate in time and space. When the ion packet of interest enters the photodissociation region, it is irradiated by a pulsed (12-ns duration, ArF* 193-nm) excimer laser (Questek, Inc., Billerica, MA, series 200). The laser beam is focused into the center of the photodissociation region by using a 300-mm cylindrical lens to form a narrow plane $(0.025 \mathrm{~cm} \times 1.0 \mathrm{~cm})$ perpendicular to the flight path of the ions. Extreme care is exercised to ensure that the laser beam does not strike metal surfaces as it passes through the photodissociation region. The laser pulse energy is measured by placing a pyroelectric energy meter (Scientech, Boulder, CO, Vector D200) in the excimer beam path prior to passage through the final focusing lens and high vacuum quartz window on the photodissociation region. The measured value is corrected for attenuation by the final optics by using a factor determined by breaking vacuum and measuring the laser pulse energy after passage through the final focusing lens and high vacuum quartz window.

The timing for firing the 193-nm pulse is controlled by a digital delay generator (EG \& G Princeton Applied Research, Princeton, NJ, Model 9650), which receives a start trigger from a photodiode positioned to detect a reflection of the 337-nm desorbing laser pulse. The necessary delay time setting between the desorption and photodissociation laser pulses for various mass analyte ions was established in two ways. Initially, the instrument was modified to position a microchannel plate detector in the ion flight path at the photodissociation point, and flight times for various mass ions were measured directly under comparable acceleration conditions. These measured flight times were subsequently corrected for the delay between triggering and firing of the excimer laser $(+1.365 \mu \mathrm{s})$ to establish the time delay setting for the digital delay generator. The microchannel plate detector was then removed and pulsed photodissociation was performed 
in separate experiments. In subsequent experiments it was found that a simple calculation of ion flight times from first principles that used measured values for the instrumental conditions gave sufficiently accurate values for the time delay setting. Final optimized overlap of the photodissociation laser pulse and the ion packet of interest is easily achieved by making small adjustments in the calculated time delay value and observing the photodissociation response in the recorded ion signals. Jitter in the delay timing between the two laser pulses was found to be no greater than $\pm 2.0 \mathrm{~ns}$.

Time-of-flight spectra are recorded via a LeCroy (Chestnut Ridge, NY) 9450A digital storage oscilloscope. The start trigger for spectrum acquisition by the oscilloscope is provided by the photodiode positioned to detect a reflection of the 337- $\mathrm{nm}$ desorbing laser pulse. All reported spectra are acquired from 50 averaged laser shots. The experimental time delay between the desorption and photodissociation laser pulses also can be measured with the LeCroy oscilloscope by monitoring the output of a photodiode positioned to detect the 193-nm laser pulse after it exits the photodissociation region through a second high vacuum quartz window. Following spectrum acquisition, the data are transferred via a GPIB interface to a personal computer for analysis and/or mass calibration via the GRAMS/386 v1.2 software (Galactic Industries Corp., Salem, NH).

Sample preparation was performed by dissolving the analyte and matrix in separate solutions of methanol or $0.1 \%(\mathrm{v} / \mathrm{v})$ trifluoroacetic acid and methanol. Two-microliter aliquots of each solution were applied to a 3-mm-diameter stainless steel probe tip and allowed to air dry to form a solid crystalline deposit. The resulting matrix-to-analyte ratio typically ranged from 100:1 to $1000: 1$ and was optimized individually to give the highest yield of analyte ions. The probe was then inserted through a high vacuum inter- lock into the source region of the mass spectrometer and positioned so that the probe tip protruded through a hole drilled in the center of the TOFMS repeller electrode. The 337-nm output of a pulsed $\mathrm{N}_{2}$ laser (Laser Science Inc., Newton, MA, VSL-337ND; pulse duration $5 \mathrm{~ns}$ ) was focused by a $350-\mathrm{mm}$ fused silica lens onto the surface of the probe tip at an angle of $60^{\circ}$ relative to the surface normal to form an elliptical spot of $\sim 0.0056 \mathrm{~cm}^{2}$. Desorbing laser irradiation was controlled by using a variable attenuation, rotatable neutral density wheel (Oriel, Stratford, CT, Model 28650) to optimize production of analyte ions. Typical desorbing laser irradiation ranged from 8.6 to $10.7 \mathrm{~mJ} / \mathrm{cm}^{2}$.

MALDI TOF spectra and MALDI-193-nm photodissociation TOF spectra were acquired for bradykinin $\left(M_{\mathrm{r}}=1059.6 \mathrm{Da}\right)$, melittin $\left(M_{\mathrm{r}}=2845.7 \mathrm{Da}\right)$, bovine insulation $\left(M_{\mathrm{r}}=5733.4 \mathrm{Da}\right)$, and lysozyme $\left(M_{\mathrm{r}}=\right.$ 14,305.1 Da). All peptides and proteins were used as purchased (Sigma Chemical Co., St. Louis, MO). the matrix $\alpha$-cyano-4-hydroxycinnamic acid also was used as purchased (Aldrich Chemical Co., Milwaukee, WI).

\section{Results}

Figure $2 a-c$ shows partial TOF spectra of the nonapeptide bradykinin taken with TOF-I biased at voltages of $0 \mathrm{~V},+3.23 \mathrm{kV}$, and $+5.33 \mathrm{kV}$. The repeller voltage was held constant at $+15 \mathrm{kV}$ and TOF- $\Pi$ was fixed at $0 \mathrm{~V}$. Figure $2 \mathrm{a}$ is identical to a conventional single stage acceleration MALDI TIF spectrum of bradykinin with the $[\mathrm{M}+\mathrm{H}]^{+}$signal observed at a flight time of 19.800 $\mu \mathrm{s}$. As the voltage applied to TOF-I is increased to $+3.23 \mathrm{kV}$ and then $+5.33 \mathrm{kV}$ (Figure $2 \mathrm{~b}$ and $2 \mathrm{c}$, respectively), the $[\mathrm{M}+\mathrm{H}]^{+}$ion signal separates into several distinct peaks. The $[\mathrm{M}+\mathrm{H}]^{+}$ion signals shift to longer flight times $(20.813 \mu \mathrm{s}$ at $+3.23 \mathrm{kV}$ and $21.814 \mu \mathrm{s}$ at $+5.33 \mathrm{kV}$ ) because of the lower total acceleration out of the ion source. Additional peaks

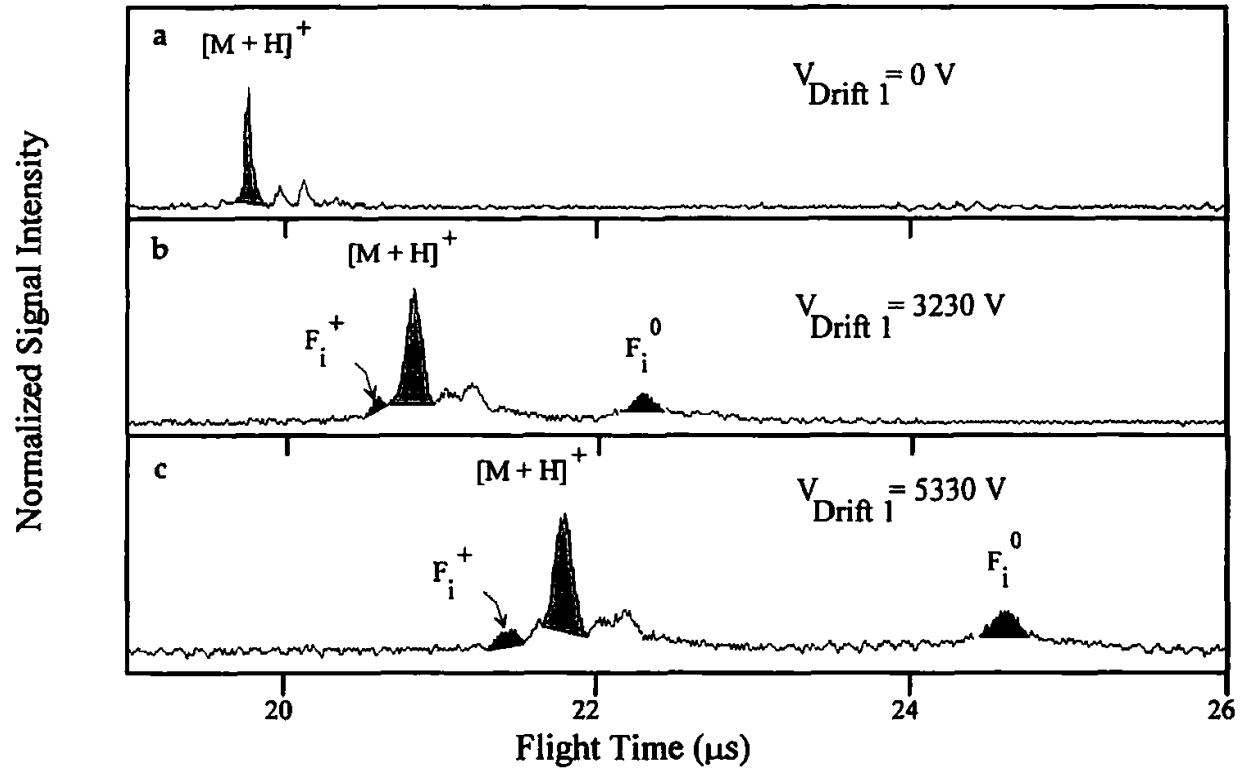

Figure 2. Partial TOF spectra showing the $[\mathrm{M}+\mathrm{H}]^{+}$region for the peptide bradykinin taken with the first drift region biased at (a) $0 \mathrm{~V}$, (b) $+3.23 \mathrm{kV}$, and (c) $+5.33 \mathrm{kV}$. Separation of the [M $+\mathrm{H}]^{+}$signal into component signals representing intact $[M+$ $\mathrm{H}]^{+}$and metastable ion decay product species $\mathrm{F}_{i}^{+}$and $\mathrm{F}_{i}^{0}$ is observed as the voltage applied to the first drift region is increased. 
arise as a result of metastable decay (unimolecular decay and/or CID) of bradykinin $\left[\mathrm{M}+\mathrm{H}^{+}\right.$in the drift region of the TOF-I $[16,24]$. Neutral metastable decay products $F_{i}^{0}$ are shifted to longer flight times relative to the $[\mathrm{M}+\mathrm{H}]^{+}$ions because they do not experience the second stage acceleration. Ionic metastable decay products $\mathrm{F}_{i}^{+}$are accelerated by the second stage field, but because of their lower masses, arrive at shorter flight times than the bradykinin [M + $\mathrm{H}^{+}$ions. It must be emphasized, however, that for the species $[\mathrm{M}+\mathrm{H}]^{+}, \mathrm{F}_{i}^{0}$ and $\mathrm{F}_{\mathrm{i}}^{+}$arrive at the photodissociation region at the same time and separation of the species only occurs as a result of the second stage of acceleration and upon traversing TOF-II.

Figure 3 shows partial two-stage TOF spectra of bradykinin taken with the 193-nm photodissociation laser blocked (Figure 3a) and with the photodissociation laser unblocked (Figure $3 b$ ). These spectra were acquired with the ion repeller biased at $+15 \mathrm{kV}$ and TOF-I biased at $+5.0 \mathrm{kV}$. The signals at 21.588 and $24.209 \mu$ s correspond to bradykinin $\left[\mathrm{M}+\mathrm{H}^{+}\right.$and $\mathrm{F}_{\mathrm{i}}^{0}$ ions, respectively. A signal from $\mathrm{F}_{i}^{+}$ions also is observed at a flight time of $21.276 \mu \mathrm{s}$. With the 193-nm photodissociation laser unblocked (Figure $3 \mathrm{~b}$ ) a portion of the $[\mathrm{M}+\mathrm{H}]^{+}$ions within the focal volume of the laser pulse are photodissociated to form product ions (denoted as photofragment ions). The lower mass photofragment ions are separated from the $[\mathrm{M}+\mathrm{H}]^{+}$ ions by the second stage of acceleration and upon traversing TOF-II, yield the pronounced dip (photodepletion) in the $[\mathrm{M}+\mathrm{H}]^{+}$ion signal. The assignment of this signal to photodepletion was confirmed by making small adjustments ( $\pm 20-40 \mathrm{~ns})$ in the delay time for firing of the excimer laser and observing equivalent time shifts in the position of the photodepletion signal. At an irradiance of $82-\mathrm{mJ} / \mathrm{cm}^{2}$ saturation occurs and we estimate that $76 \%$ of the ions present in the laser focal volume dissociate while traversing the $0.5 \mathrm{~cm}$ prior to TOF-II. The width of the photodepletion signal is determined by the laser pulse width (12 ns), the width of the laser beam $(0.025 \mathrm{~cm}$ in the direction of the ion flight path) at the laser beam and ion beam intersection, the laboratory velocity of the ions, and the distribution of ion translational energies that make up the irradiated ion packet. The temporal and spatial profile of the ion packet increases in time due to the distribution of ion translational energies. The increased temporal and spatial profile of the ion packet results in broadening of the photodepletion signal as the total flight time of ions increases.

A photodepletion signal for $F_{i}^{0}$ also is observed in the spectrum shown in Figure $3 \mathrm{~b}$. This signal must result from 193-nm photoionization of $\mathrm{F}_{i}^{0}$ to yield ionized species that are subjected to the second stage acceleration field and separate from the neutral species in TOF-II. Photodepletion of $\mathrm{F}_{\mathrm{i}}^{+}$also is observed. This signal must result from 193-nm photodissociation of $\mathrm{F}_{i}^{+}$to produce lower mass-to-charge ratio photofragment ions and additional neutral molecules. The photofragment ions separate from the $\mathrm{F}_{i}^{+}$signal after the second stage acceleration and upon traversing TOF-II. In addition, new ion signals are observed between the $[\mathrm{M}+\mathrm{H}]^{+}$ion signal and $\mathrm{F}_{\mathrm{i}}^{0}$ signal. These new ion signals are attributed to field ionization of excited neutral species in the second acceleration field [25]. The excited neutral species are probably formed by single photon excitation of the $F_{i}^{0}$ to sufficiently high energy levels that field ionization is a facile process. Field ionization of photoexcited neutrals [26] would yield ions that have less than the total acceleration; thus the flight times for these ions would fall between the $[\mathrm{M}+\mathrm{H}]^{+}$ions and $\mathrm{F}_{\mathrm{i}}^{0}$.

Two-stage TOFMS-193-nm photodissociation was performed on melittin, bovine insulin, and lysozyme analyte ions produced by MALDI. Examples of photodissociation laser "on" TOF mass spectra obtained
Figure 3. Partial TOF spectra showing $[\mathrm{M}+\mathrm{H}]^{+}$region for the peptide bradykinin taken with $193 \mathrm{~nm}$ photodissociation laser (a) blocked and (b) unblocked. Photodissociation of the [M+ $\mathrm{H}^{+}$ions at a laser irradiance of $82 \mathrm{~mJ} / \mathrm{cm}^{2}$ yields a pronounced photodepletion of the detected ion signal resulting from the production of lower mass photofragments. Photodepletion is also observed in the signals for the metastable ion decay product species $\mathrm{F}_{i}^{+}$and $\mathrm{F}_{i}^{0}$ as a result of $193 \mathrm{~nm}$ photodissociation and photoionization, respectively, of these species.

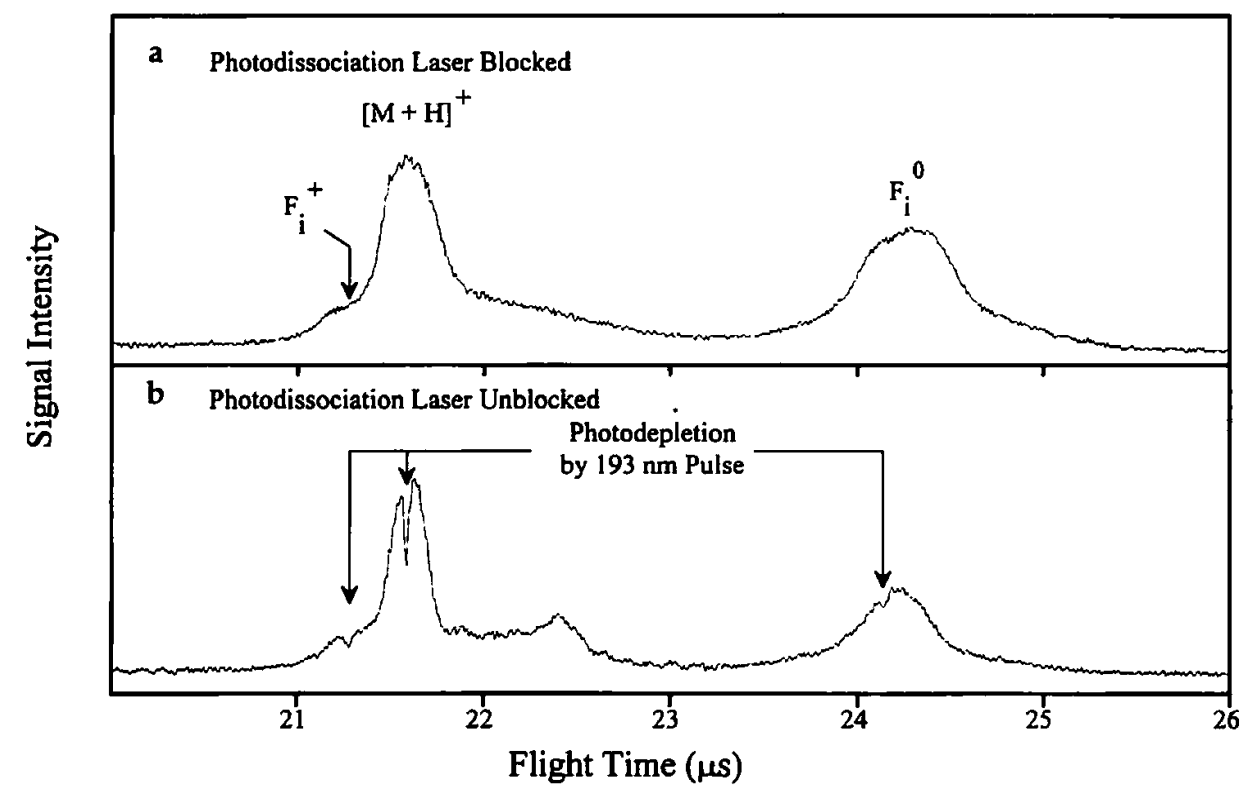


for these analytes are shown in Figures $4 a-c$. For both the singly charged melittin and bovine insulin ions a pronounced 193-nm photodepletion signal was obtained at a laser irradiance of $12 \mathrm{~mJ} / \mathrm{cm}^{2}$ and saturation of the signal occurs at somewhat higher values. Clearly, 193-nm photodissociation of analyte ions as large as bovine insulin is readily achieved. Conversely a photodepletion could not be observed for singly charged lysozyme at a 193-nm laser irradiance of 100 $\mathrm{mJ} / \mathrm{cm}^{2}$; however, a 193-nm photodepletion signal was obtained for the doubly charged lysozyme (see Figure 4c) and triply charged ions at a laser irradiance of 18 $\mathrm{mJ} / \mathrm{cm}^{2}$.

The relative photodepletion signals were measured as a function of 193-nm pulse energy at fixed first and second stage acceleration conditions to probe the number of 193-nm photons that lead to analyte dissociation. If dissociation is the only means of ion deactivation, $d\left(\sum I_{[\mathrm{M}+\mathrm{H}]^{+}}\right) / d t=\left(\sigma_{1}, \sigma_{2}^{*} \cdots \sigma_{n}\right)^{*}\left(F^{n}\right)^{*}$ $\left(\sum I_{[\mathrm{M}+\mathrm{H}]^{+}}\right)$where $\sigma_{1}, \sigma_{2}, \cdots, \sigma_{n}$ are the 193-nm absorption cross sections for the absorption of 1 to $n$ photons, $F$ is the laser photon flux, and $\sum I_{[\mathrm{M}+\mathrm{H}]^{+}}$is the total ion current (i.e., number of parent ions irradiated). Because the time available for analyte photodissociation is fixed by the velocity of the ions in the first drift region and the distance from the point of photoactivation to the entrance of the second acceleration region, $d\left(\sum I_{[M+H]^{+}}\right) / d t$ may be approximated as the area of the photodepletion signal. Thus, a plot of $\log$ relative photodepletion signal $\left(d\left(\sum I_{[\mathrm{M}+\mathrm{H}]^{+}}\right) / d t / \sum I_{[\mathrm{M}+\mathrm{H}]^{+}}\right)$ versus log photon flux gives a line with a slope of $n$, where $n$ is the number of photons absorbed, and an intercept of $\log \left(\sigma_{1}^{*} \sigma_{2}^{*} \cdots \sigma_{n}\right)$.

Figure $5 \mathrm{a}$ shows the results of laser power studies for the photodissociation of bradykinin $[\mathrm{M}+\mathrm{H}]^{+}$, singly charged bovine insulin, and doubly charged lysozyme. The points along the vertical axis represent the relative photodepletion of the $[\mathrm{M}+\mathrm{H}]^{+}$ion signal. These val- ues are obtained by generation of a difference signal first. The background signal (193 nm blocked) was subtracted from each two-stage photodissociation spectrum to yield a spectrum that contained only the photodepletion signals. The curve fitting feature of the GRAMS/386 software was then used to obtain the integrated area of the photodepletion signals. A linear regression of the log relative photodepletion signal versus log laser flux for each analyte gave a slope 0.90 for bradykinin ( $R=0.88), 0.91$ for bovine insulin ( $R=$ 0.89 ), and 1.12 for doubly charged lysozyme $(R=0.89)$. Figure $5 \mathrm{~b}$ compares photodepletion of $\mathrm{F}_{i}^{0}$ with photodepletion of $[\mathrm{M}+\mathrm{H}]^{+}$for MALDI-produced bradykinin ions as a function of 193-nm laser flux. A linear regression of the $\log$ relative photodepletion signal versus log laser flux for $F_{i}^{0}$ photoionization gives a slope of $1.70(R=0.94)$.

\section{Discussion}

It is clear from the results presented that the experimental methodology for performance of photodissociation with tandem TOFMS for both fundamental studies and applications is feasible. Synchronization of the desorbing and photodissociation laser has been achieved for ions that have $12.5 \mathrm{keV}$ kinetic energies with a precision of \pm 4 ns. Simple calculations of ion flight times from first principles by using measured instrumental parameters combined with a knowledge of the laser and electronic triggering delays allows the photodissociation time delay setting to be established easily. The success of this approach is established by the photodepletion response observed in the TOF signals. Furthermore, the relatively low 193-nm laser irradiance required to saturate photodissociation of the analyte ions suggests that increasing the laser energy while defocusing the laser across the ion flight path will allow a large fraction ( $50 \%$ or more) of the total

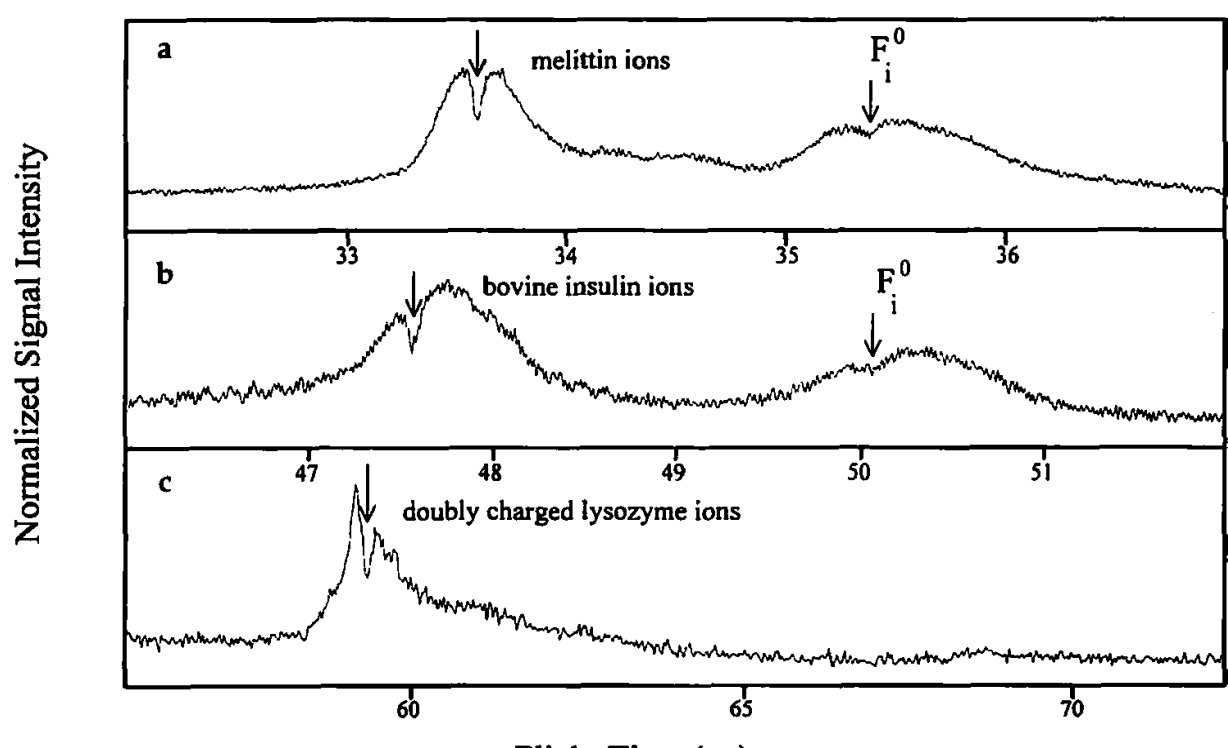

Flight Time ( $\mu \mathrm{s})$
Figure 4. Partial TOF spectra showing the $193 \mathrm{~nm}$ photodissociation of the (a) singly charged melittin ion $\left(82 \mathrm{~mJ} / \mathrm{cm}^{2}\right)$, (b) singly charged bovine insulin ion $\left(82 \mathrm{~mJ} / \mathrm{cm}^{2}\right)$, and $(\mathrm{c})$ the doubly charged lysozyme ion (104 $\left.\mathrm{mJ} / \mathrm{cm}^{2}\right) .193 \mathrm{~nm}$ photodissociation of the intact ions is observed in all cases as evidenced by the photodepletion dip in the detected ion signals. $193 \mathrm{~nm}$ pho toionization of the metastable ion decay product neutrals $\mathrm{F}_{i}^{0}$ is also observed for melittin and bovine insulin. 
Figure 5. (a) Log-log plot of the relative photodepletion signal as a function of $193 \mathrm{~nm}$ photon flux for photodissociation of $\downarrow$, lysozyme $\mathrm{M}^{2+}, \mathrm{\square}$, bovine insulin $\mathrm{M}^{+}$, and $\Delta$, bradykinin $\left[\mathrm{M}+\mathrm{H}^{+}\right.$. (b) Photodissociation of $\Delta$, bradykinin [M $+\mathrm{H}]^{+}$ions, is compared to photoionization of $\boldsymbol{\square}$, bradykinin metastable ion decay product neutrals $F_{i}^{0}$.
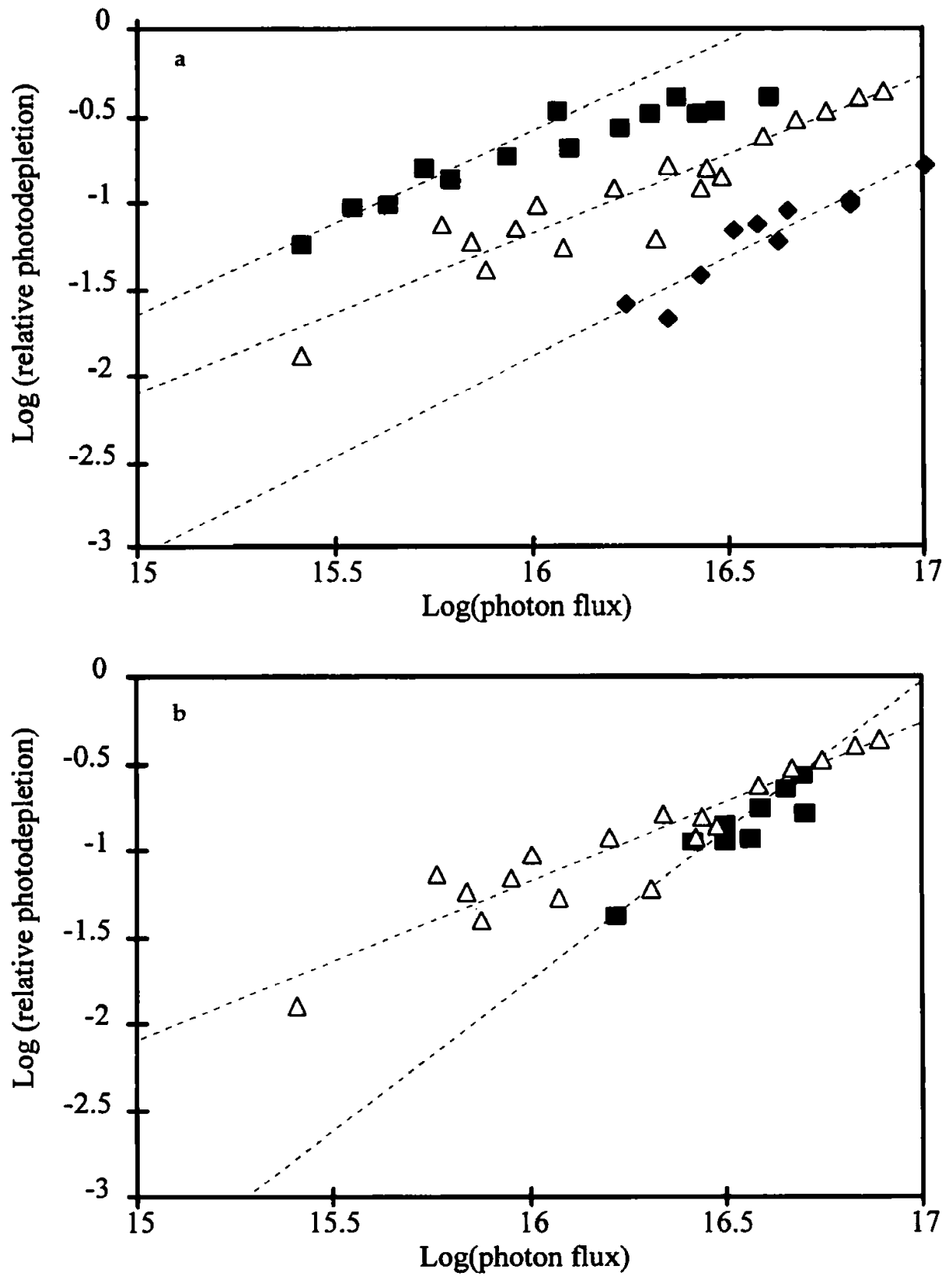

ion population to be photoactivated. Conversely, a technique such as postsource pulsed focusing [27] could be utilized to compress the ion packet and thus increase the numbers of ions irradiated.

The successful implementation of the laser photodissociation experimental methodology allows us to address the two additional goals of these experiments; namely, what combination of ion dissociation processes will contribute to the photofragment ion spectrum and what are the limitations to photodissociation of high molecular weight ions produced by MALDI. With regard to the first question, it is clear that a variety of analyte dissociation processes occur in the photodissociation experiments described. Product ions are produced by $193-\mathrm{nm}$ photodissociation of the ana- lyte $[\mathrm{M}+\mathrm{H}]^{+}$ion and by metastable decay. Subsequent 193-nm photodissociation of the metastable ion decay product ions $\mathrm{F}_{i}^{+}$also leads to product ion formation, and product ions arise from 193-nm photoionization of the metastable decay product neutrals $F_{i}{ }^{0}$. Therefore, analysis of the photofragment spectrum requires that the product ions observed in the TOF spectrum be associated with the specific processes that lead to their production. Subtraction of the $\mathrm{F}_{1}^{+}$and $\mathrm{F}_{i}{ }^{0}$ spectra acquired with the photodissociation laser blocked provides only a partial resolution of the question of product ion origin because photoionization of $\mathrm{F}_{1}^{0}$ and photodissociation of $\mathrm{F}_{\mathrm{i}}^{+}$are not taken into account in this approach. Identification of the contribution from photoionized $F_{i}^{0}$ could be accomplished by 
placing a strong retarding field in front of the photodissociation region, thereby separating $\mathrm{F}_{\mathrm{i}}^{0}$ and $[\mathrm{M}+$ $\mathrm{H}^{+}$and $\mathrm{F}_{\mathrm{i}}^{+}$ions, and recording the 193-nm photoionization spectrum. Subsequently, the $F_{i}^{0}$ photoionization spectrum could be subtracted from the photofragment ion spectrum to remove the contribution that arises from this source. No simple approach is available, however, to identify and eliminate the contribution from photodissociation of $\mathrm{F}_{i}^{+}$. Ultimately, separation of the $[\mathrm{M}+\mathrm{H}]^{+}$ions from all metastable product species (via a retarding potential or other type of energy analyzer) immediately prior to performing laser photodissociation may be necessary to obtain a photofragment ion spectrum that results solely from $[\mathrm{M}+\mathrm{H}]^{+}$laser photodissociation.

With regard to photodissociation of high molecular weight analyte ions, our results clearly demonstrate that analytes as large as singly charged bovine insulin undergo photodissociation on time scales compatible with TOF analysis. The rate for 193-nm photodissociation of bovine insulin must exceed $4 \times 10^{6} \mathrm{~s}^{-1}$ for the photodepletion signal to be observed in the two-stage TOFMS. This minimum rate is based on the velocity of the ions in the first drift region and the distance 0.5 $\mathrm{cm}$ ) between the point of photoactivation and the second acceleration stage. Similar calculations place lower limits on the dissociation rates for melittin and bradykinin ions at $6 \times 10^{6}$ and $9 \times 10^{6} \mathrm{~s}^{-1}$, respectively. Furthermore, we suggest that the 193-nm photodissociation signal observed for bovine insulin results from dissociation into structurally significant ions (not simply loss of $\mathrm{H}_{2} \mathrm{O}$ or $\mathrm{NH}_{3}$ ). For example, for the photofragment ions formed on the long flight time edge of the excimer laser pulse to separate from the undissociated singly charged ions, the photofragment ion mass-to-charge ratio can be no more than the $96 \%$ $(5504 \mathrm{u})$ of the mass of singly charged bovine insulin.

The integer slope of 1 obtained from the 193-nm photodissociation power studies (Figure $5 \mathrm{a}$ ) of the [M $+\mathrm{H}]^{+}$ions is consistent with photodissociation due to the absorption of a single 193-rm photon ( $h \nu=6.42$ $\mathrm{eV}$ ). We would expect the ionization potential for the neutral products to be $>6.4 \mathrm{eV}$ (Figure 5b). A slope of 2 for $F_{i}^{0}$ photoionization is consistent with the expectation that the 193-nm two photon energy $(2 h \nu=12.8$ $\mathrm{eV}$ ) is sufficient to exceed the ionization energy for the neutral products. The difference in the slopes for the two processes validates the results of the power studies, and we are confident that photodissociation is, in fact, a one photon process. Dissociation rates that exceed $10^{6} \mathrm{~s}^{-1}$ at photoactivation energies of $6.4 \mathrm{eV}$ for bovine insulin are difficult to reconcile with the arguments presented by Griffin and McAdoo [13]. Either the internal energy of the ions formed by MALDI is high (and mass dependent) or the dissociation threshold $E_{0}$, is much less than the $100 \mathrm{~kJ} / \mathrm{mol}$ proposed by Griffin and McAdoo. Alternatively, the basic assumptions of statistical theory may not be applicable to high molecular weight ion photodissociation. Clearly, fur- ther studies in this area are warranted. In particular, it would be interesting to compare photodissociation of MALDI- versus ESI-formed ions as well as photodissociation of MALDI-formed ions that have been collisionally relaxed.

The observation that singly charged lysozyme ions do not photodissociate and the doubly $(m / z 7153 \mathrm{Da})$ and triply charged ions $(m / z 4768 \mathrm{Da})$ do is rather interesting. We have considered several interpretations in an effort to explain this observation. First, the difference in photodissociation efficiency may reflect charge state dependent differences in MALDI ion internal energy. Photoactivation of a "hotter" ion would allow the dissociation rate to exceed the minimum (singly charge lysozyme $>3 \times 10^{6} \mathrm{~s}^{-1}$; doubly charged lysozyme $>4 \times 10^{6} \mathrm{~s}^{-1}$ ) for observation of the photodepletion signal. We note, however, that the degree of metastable decay of both the singly and doubly charged species, as measured by the relative production of $\mathrm{F}_{i}^{0}$ in retarding field experiments, is identical, an observation inconsistent with different internal energies of the two species prior to photodissociation. Alternatively, the activation threshold $\left(E_{0}\right)$ for doubly charged lysozyme might be substantially lower than for singly charged lysozyme due to Coulombic repulsion and/or charge state dependent ion conformation. If this were true, however, it would again be expected that doubly charged species would undergo substantially more metastable dissociation than the singly charged species, but this behavior is not observed.

Less efficient activation of the singly charged lysozyme ion at $193 \mathrm{~nm}$ (whether via poorer absorption or more efficient nondissociative relaxation) as a result of charge state influence on the protein electronic structure might also explain the inability to photodissociate the singly charged ion. The intercept of the $\log -\log$ power studies (Figure 5a) gives, to a first approximation, the absorption cross section $\sigma$ for the ions at $193 \mathrm{~nm}$. Singly charged bovine insulin ions have the largest cross section $\left(\sigma=9 \times 10^{-16} \mathrm{~cm}^{2}\right)$ followed by bradykinin ions $\left(\sigma=2 \times 10^{-16} \mathrm{~cm}^{2}\right)$ and the doubly charged lysozyme ions $\left(\sigma=1 \times 10^{-20}\right.$ $\mathrm{cm}^{2}$ ). The small $\sigma$ value obtained for the doubly charged lysozyme ions is surprising given the increased number of 193-nm absorbing amide bonds. This result truly may reflect poorer absorption at 193 $\mathrm{nm}$ by the lysozyme ions or it may reflect the influence of increasing numbers of nondissociative pathways. For example, if nondissociative relaxation pathways are competitive with dissociative pathways for the photoexcited ions, the $\sigma$ values determined from the intercept will reflect the ion absorption cross section and the rate for the nondissociative processes, for example, a fast radiative decay process will cause the $\sigma$ value to appear artificially small because many more ions must be excited to observe the photodepletion signal. However, in either case, no clear rationale exists to explain why singly and doubly charged 
lysozyme ions should exhibit substantially different 193-nm photodissociation behavior. Further investigations of wavelength dependent ion absorption cross sections and absolute rates of ion photodissociation are needed to interpret the charge dependent lysozyme photodissociation behavior.

\section{Conclusions}

From these preliminary experiments it is clear that tandem TOF-photodissociation of peptide and protein ions produced by MALDI can be accomplished. For example, current instrumentation and timing hardware are available to synchronize the photodissociation laser pulse with the packet of analyte ions as they travel at high velocities through TOF-I. Rather modest photodissociation laser irradiance $\left(<20 \mathrm{~mJ} / \mathrm{cm}^{2}\right)$ is needed for dissociation of singly charged bradykinin, melittin, bovine insulin, and doubly charged lysozyme ions. The experiments reported here clearly show that the photochemistry of metastable fragments contributes to the photofragment ion spectrum. Currently we are developing methods to eliminate product ions that do not originate from intact parent ion photodissociation. These approaches include new matrices that produce "cooler" $[\mathrm{M}+\mathrm{H}]^{+}$ions, the use of electrostatic fields to separate metastable ions from stable $[\mathrm{M}+\mathrm{H}]^{+}$ions prior to photodissociation, and the development of methods for mass analysis of the photofragment ion spectrum. A second generation photodissociation apparatus that incorporates a twostage gridded reflectron for mass analysis of the photofragment ions and the results of these experiments are the subject of a future paper (Gimon-Kinsel, M. E.; Kinsel, G. R.; Russell, D. H., in preparation).

Laser power studies suggest that photodissociation occurs as the result of the absorption of a single 193-nm photon and dissociation of the analyte ions occurs with rates greater than $10^{6} \mathrm{~s}^{-1}$. The ability to dissociate ionized proteins as large as bovine insulin $(\mathrm{m} / \mathrm{z}$ 5734) and doubly charged lysozyme $(m / z 7153)$ with a single 6.42-eV photon suggests possibilities for structural mass spectrometry of rather large biomolecules. The inability to photodissociate singly charged lysozyme ions $(m / z 14,306)$ under the identical conditions that allow photodissociation of doubly charged ion presents a puzzle for which the experimental results provide no clear explanation. Additional studies are needed to explore the influence of molecule size, ion charge state, and laser photodissociation wavelength on absolute MALDI ion dissociation rates.

\section{Acknowledgments}

Funding for this research was provided by the U.S. Department of Energy, Division of Chemical Sciences, Office of Basic Energy Science (DE-FG05-89ER75502). M.E.G. also thanks Dow Chemical USA for its financial support through an ACS Division of Analyt- ical Chemistry Graduate Fellowship and the W. R. Grace Foundation for its financial support through a graduate fellowship.

\section{References}

1. (a) Karas, M.; Bahr, U.; Giessmann, U. Mass. Spectrom. Rev. 1991, 10, 335; (b) Chait, B. T.; Kent, S. B. H. Science 1992, 257, 1885.

2. Hillenkamp, F.; Karas, M.; Beavis, R. C.; Chait, B. T. Anal. Chem. 1991, 63, 1193A.

3. Biemann, K.; Martin, S. A. Mass Spectrom. Rev. 1987, 6, 1; (b) Jensen, N. J.; Gross, M. L. Mass Spectrom. Rev. 1987, 7, 1.

4. (a) Gross, M. L., Cerny, R. L.; Giblin, D. E.; Rempel, D. L.; MacMillan, D. K.; Hu, P.; Holliman, C. L. Anal. Chim. Acta 1991, 250, 105; (b) Gross, M. L. in Methods in Enzymology, Vol. 193; McCloskey, J. A., Ed; Academic Press; San Diego, 1990; $\mathrm{p} 131$.

5. (a) Shukla, A. K.; Futrell, J. H. in Topics in Mass Spectrometry, Vol. 1: Experimental Mass Spectrometry; Russell, D. H., Ed.; Plenum Press: New York, 1994; pp 71-112; (b) Cooks, R. G. Collisional Spectroscopy; Plenum Press: New York, 1978.

6. Cooks, R. G.; Ast, T.; Mabud, A. Int. J. Mass Spectrom. Ion Processes 1990, 100, 209.

7. (a) Tecklenburg, R. E.; Russell, D. H. Mass Spectrom. Rev. 1990, 9, 405-451; (b) Harris, F. M.; Beynon, J. H. in Gas Phase Ion Chenistry, Vol. 3; Bowers, M. T., Ed.; Academic Press: Orlando, 1984; pp 99-128; (c) Dunbar, R. C. in Gas Phase Ion Chemistry, Vol. 3; Bower, M. T. Ed.; Academic Press: Orlando, 1984; pp 129-165.

8. (a) Price, D.; Milnes, G. J. Int. J. Mass Spectrom. Ion Processes, 1990, 99, 1-39; (b) Cotter, R. J. Anal. Chem. 1992, 64, $1027 \mathrm{~A}-1039 \mathrm{~A}$.

9. (a) Köster, C.; Castoro, J. A.; Wilkins, C. L. J. Am. Chem. Soc. 1992, 114, 7572; (b) Solouki, T.; Gillig, K. J.; Russell, D. H. 1994, 66, 1583; (c) Campana, J. Presented at the 42nd ASMS Conference on Mass Spectrometry and Applied Topics, Chicago, IL, 1994, p 886.

10. (a) Pinkston, J. D.; Rabb, M.; Watson, J. T.; Allison, J. Rev. Sci. Instrum. 1986, 57, 583; (b) Liu, Y.; Zhang. Q.-L.; Tittel, F. K.; Curl, R. F.; Smalley, R. E. J. Chem. Phys. 1986, 85, 7434; (c) Alexander, M. L.; Levinger, N. E.; Johnson, M. A.; Ray, D.; Lineberger, W. C. J. Chem. Phys. 1988, 88, 6200; (d) Weinhauf, R.; Walter, K.; Weickhardt, C.; Boesl, U.; Schlag, E. W. Z Naturforsch, 1989, 44a, 1219; (e) Boesl, U.; Weinhauf, R.; Walter, K.; Weickhardt, C.; Schlag, E. W. Ber Bunsenges. Phys. Chem. 1990, 94, 1357; (f) Laihing, K.; Cheng, P. Y.; Taylor, T. G.; Willey, K. F.; Peschke, M.; Duncan, M. A. Anal. Chem. 1989, 61, 1460; (g) Cornett, D. S.; Duncan, M. A.; Amster, I. J. Presented at the 39th ASMS Conference on Mass Spectrometry and Allied Topics, Nashville, TN 1991, pp 146-147; (h) Comett, D. S.; Peschke, M. LaiHing, K.; Cheng, P. Y.; Willey, K. F.; Duncan, M. A. Rev. Sci. Instrum. 1992, 63, 2177; (i) Cornish, T.; Cotter, R. J. Rapid Commun. Mass Spectrom. 1992, 6, 242; (j) Cornish, T. J.; Cotter, R. J. Anal. Chem. 1993, 65 , 1043; (k) Seeterlin, M. A.; McLane, R. D.; Vlasak, P. R.; Beussman, D. J.; Enke, C. G. Presented at the 42nd ASMS Conference on Mass Spectrometry and Allied Topics, Chicago, IL, 1994, pp 938a-938b.

11. (a) Watson, C. H.; Baykut, G.; Eyler, J. R. Anal. Chem. 1987, 59, 1133; (b) Griffin, P. R.; Kumar, S.; Shabanowitz, J.; Charonneau, H.; Namkung, P. C.; Walsh, K. A.; Hunt, D. F.; Petra, P. H. J. Biol. Chem. 1989, 264, 10966; (c) Gord, J. R.; Buckner, S. W.; Freiser, B. S. J. Am. Chem. Soc. 1989, 111, 3753; (d) Snodgrass, J. T.; Dunbar, R. C.; Bowers, M. T. J. Phys. Chem. 1990, 94, 3648; (e) Gorman, G. S.; Cornett, D. S.; Amster, I. J. Presented at the 39th ASMS Conference on Mass 
Spectrometry and Allied Topices, Nashville, TN, 1991, pp 489-490; (f) Castoro, J. A.; Nuwaysir, L. M.; ljames, C. T.; Wilkins, C. L. Anal. Chemt. 1992, 64, 2238-2243; (g) Solouki, T.; Russell, D. H. Appl. Spectrosc, 1993, 47, 211-217; (h) Senko, M. W.; Beu, S. C.; McLafferty, F. W. Anal. Chem. 1994. $66,415$.

12. (a) Robinson, P. J.; Holbrook, K. A. Unimolecular Reactions; Wiley: New York, 1972; (b) Rosenstock, H. M.; Wallenstein, M. B.; Wahrhaftig, A. L.; Eyring, H. Proc. Natl. Acad. Sci. U.S. A. 1952, 38, 667 .

13. Griffin, L. L.; McAdoo, D. J. J. Am. Soc. Mass Spectrom. 1993, $4,11$.

14. Hunt, D. F.; Shabanowitz, J.; Yates, J. R. J. Chem. Soc., Chem. Commun. 1987, 548.

15. Little, D. P.; Speir, J. P.; Senko, M. W.; O'Connor, P. B.; McLafferty, F. W. Anal. Chem. 1994, 66, 2809.

16. (a) Martin, S. A.; Hill, J. A.; Kitrell, C.; Biemann, K. J. Am. Soc. Mass Spectrom. 1990, 1, 107; (b) Köchling, H. J.; Hill, J. A.; Biller, J. E.; Biemann, K. Presented at the 41st ASMS Conference on Mass Spectrometry and Allied Topics, San Francisco, CA, 1993, p 435a.

17. Schlag, E. W.i Levine. R. D. I. Phys. Chem. 1992, 96, 10608.

18. Bernshtein, V.; Oref, I. I. Phys. Chem. 1994, 98, 136.
19. Preston-Schaffter, L. M. Dissertation, Texas A \& M University, 1994.

20. Zhou, J.; Ens, W; Pope-Schiemer, N.; Standing, K. G.; Westmore, J. B. Int. J. Mass Spectrom Ion Processes 1993, 126, 115.

21. Loo, J. A.; Edmonds, C. G.; Smith, R. D. Anal. Chem. 1993, 65, 425.

22. Smith, R. D.; Loo, J. A.; Barinaga, C. J.; Edmonds, C. G.; Udseth, H. R. J. Am. Soc. Mass Spectrom. 1990, 4, 53.

23. Preston-Schaffter, L. M.; Kinsel, G. R.; Russell, D. H. J. Am. Soc. Mass Spectrom. 1994, 5, 800.

24. (a) Kaufmann, R.; Kirsch, D.; Spengler, B. Int. J. Mass Spectrom. Ion Processes 1994, 131, 355, (b) Spengler, B.; Kirsch, D.; Kaufmann, R. J. Phys. Chem. 1992, 96, 9678.

25. Bordas-Nagy, J.; Holmes, J. L.; Mommers, A. A. Org. Mass Spectrom. 1986, 21, 629.

26. Zhang, X.; Smith, J. M.; Knee, J. L. J. Chem. Phys. 1992, 97. 2843.

27. (a) Kinsel, G. R.; Johnston, M. V. Int. J. Mass Spectrom, Ion Processes, 1989, 91, 157; (b) Kinsel, G. R.; Mowry, C. D.; McKeown, P. J.: Johunston, M. V. Int. I. Mass Spectrom. Ion Processes 1991, 104, 35; (c) Kinsel, G. R.; Grundwürmer, J. M.; Bönisch, M.; Grotemeyer, J. Presented at the 40th ASMS Conference on Mass Spectrometry and Allied Topics, Washington, DC, 1992, pp 8-9. 